\title{
3D Reconstruction and Porosity Study of a Hierarchical Porous Monolithic Metal Organic Framework by FIB-SEM Nanotomography
}

\author{
E. L. Solla ${ }^{1}$, L. Micheron ${ }^{2}$, P. Yot ${ }^{3}, J_{\text {Méndez }}^{1}$ and P. Horcajada ${ }^{2}$ \\ 1. Servicio de Microscopía Electrónica, CACTI, Universidade de Vigo, Vigo, Spain. \\ 2. Institut Lavoisier, Université de Versailles Saint-Quentin-en-Yvelines, Versailles, France. \\ 3. Institut Charles Gerhardt Montpellier, Montpellier, France.
}

Porous metal-organic frameworks (MOFs) belong to a fascinating class of porous crystalline materials and currently receive much attention in regard to their potential applications in strategic domains [1]. Typically obtained as crystalline powders, MOFs need to be suitably shaped for their industrial application without affecting their textural properties. An interesting recent route is the formation of aerogel [2], which can provide additional porosity. In particular, the benchmarked cubic UiO-66 solids (UiO for Oslo University) [3], built up from $\mathrm{Zr}_{6} \mathrm{O}_{4}(\mathrm{OH})_{4}$ oxoclusters and terephthalate anions bearing different functional groups, appears to be a good candidate in storage, separation, catalysis and biomedicine [4]. UiO-66 exhibits tetrahedral and octahedral cages (6 and $11 \AA)$ accessible through microporous windows ( 4-6 $\AA$ ), leading to a high porosity (BET surface area $\sim 1200 \mathrm{~m} / \mathrm{g}$; pore volume $\sim 0.47 \mathrm{~cm}^{3} / \mathrm{g}$ ) combined with a high thermal, chemical and mechanical stability.

A gel based on small and monodispersed nanoparticles $(\sim 40 \mathrm{~nm})$ of the zirconium aminoterephthalate (UiO-66_NH $\mathrm{NH}_{2}$ ) was initially prepared [5] and then dried using supercritical $\mathrm{CO}_{2}$ leading to the formation of monolithic pieces with hierarchical porosity.

In order to perform a 3D reconstruction of the hierarchical porosity of the monolithic UiO-66 $\mathrm{NH}_{2}$ material a FIB-SEM slice \& view experiment was carried out with a Dual Beam FEI Helios Nanolab 600. FIB milling with $\mathrm{Ga}+$ ions was performed at a current of $0.28 \mathrm{nA}$ at $30 \mathrm{kV}$, keeping a distance of $20 \mathrm{~nm}$ between consecutive slices. SEM images were acquired with a $5 \mathrm{kV}$ beam at a working distance of $4 \mathrm{~mm}$. Fig. 1 presents a high magnification image of the ion beam polished material face, showing the nanoparticles that comprise the gel together with interparticle pores of different sizes.

The obtained image stack was subsequently aligned and processed with FEI Amira Resolve RT in order to produce a $1.30 \times 1.08 \times 2.88 \mu \mathrm{m} 3 \mathrm{D}$ model of the porous MOF, presented on Fig. 2. Once the 3D model was obtained, it allowed performing further calculations to gather a deeper insight of the material. Therefore, a pore size distribution calculation was performed on FIJI [6] using the plugin developed by B. Münch et al. [7], yielding a total porosity of $53 \%$ and the distribution in the 0 to $50 \mathrm{~nm}$ range presented on Fig. 3.

Interestingly, when the monolithic UiO-66_ $\mathrm{NH}_{2}$ material was subjected to $\mathrm{Hg}$ intrusion (Prior the collection, sample outgassed during $15 \mathrm{~min}$. up to a P 6.5 Pa. Density and particle size distribution were determined in the pressure range from 0.1 to $200 \mathrm{MPa}$ using a Micromeritics Autopore 9240 at room temperature) and $\mathrm{N}_{2}$ adsorption porosimetry (Sample was previously outgassed at $150{ }^{\circ} \mathrm{C}$ overnight. $\mathrm{N}_{2}$ sorption isotherm at $77 \mathrm{~K}$ performed using a Berlsorp Mini-Bel Japan apparatus), a porosity of around $54 \%$ was found with a pore size distribution disclosed on Fig. 4. If we examine the variation of pore volume with respect of pore radius (dV/dR) on Fig 3, it clearly shows two abrupt jumps at nearly the 100 and $200 \AA$, respectively. Likewise, the $\mathrm{N}_{2}$ adsorption exhibited a bimodal distribution at nearly the 
same pore size values, therefore validating the calculations performed purely on the 3D reconstructed model. In conclusion, the 3D reconstruction by means of FIB-SEM nanotomography has been revealed as a highly valuable tool to estimate the textural properties of complex hierarchical porous solids.

\section{References:}

[1] H.C. Zhou, J.R. Long and O.M. Yaghi, Chem Rev 112 (2012) p. 673.

[2] M.R. Lohe, M. Rose and S. Kaskel, Chem. Commun. 28 (2009) p. 6056.

[3] J. Cavka, S. Jakobsen and U. Olsbye, J. Am. Chem. Soc. 130 (2008) p. 15850.

[4] D. Cunha et al., J. Mater. Chem. B. 1 (2013) p. 1101.

[5] F. Ragon et al., Inorg. Chem. 53 (2014) p. 2491.

[6] J. Schindelin, I. Arganda-Carreras and E. Frise, Nat. Methods 9 (2012) p. 676.

[7] B. Münch and L. Holzer, J. Am. Ceram. Soc. 91 (2008) p. 4059.

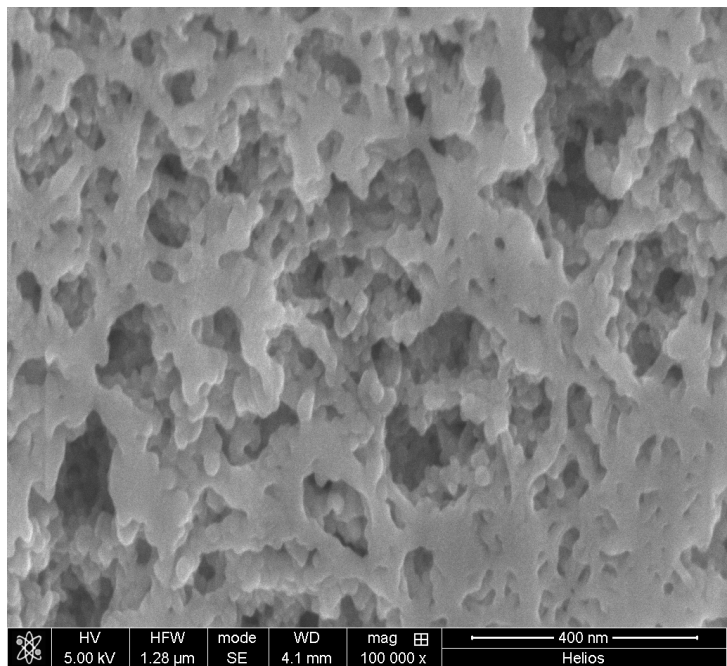

Figure 1. SEM image of the FIB polished material face.

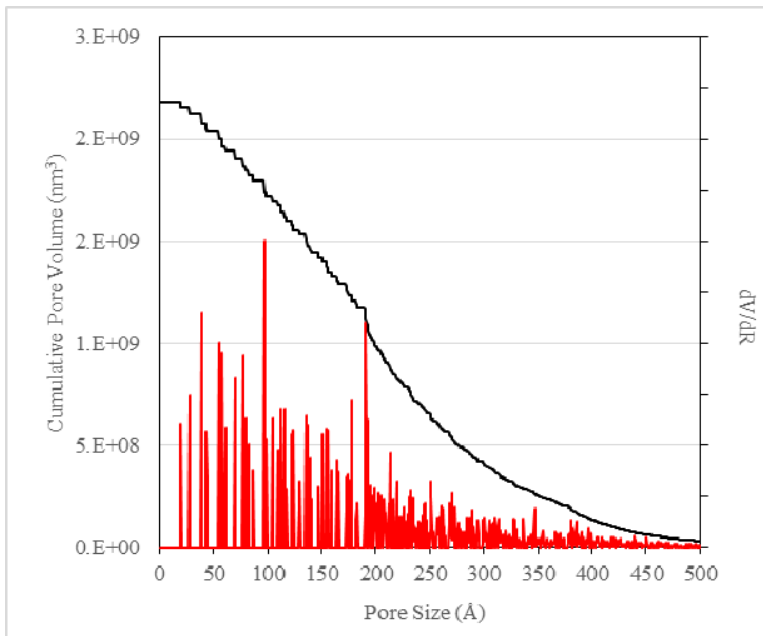

Figure 3. Pore size distribution and volume variation relative to pore radius calculated on the $3 \mathrm{D}$ structure.

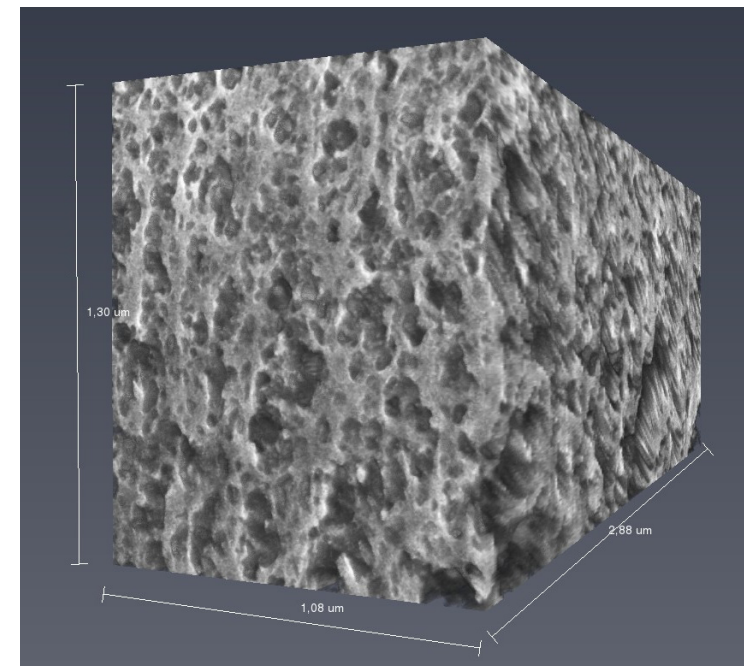

Figure 2. 3D Model of the porous MOF, reconstructed with the FIB-SEM image stack.

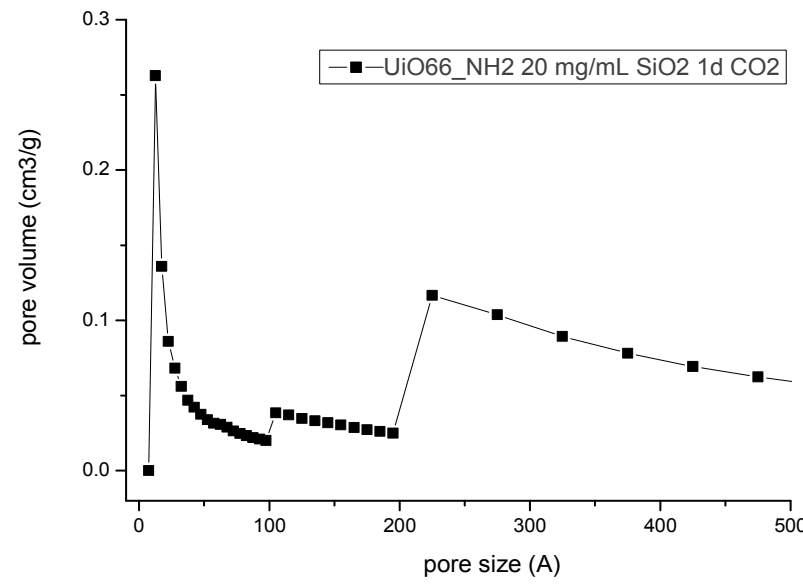

Figure 4. Pore size distribution obtained from the $\mathrm{N}_{2}$ adsorption performed on the MOF. 